\title{
EFECTO DEL OXÍGENO DISUELTO SOBRE LA BIODEGRADABILIDAD DE BIORRESIDUOS
}

\section{DISSOLVED OXYGEN EFFECT ON THE BIODEGRADABILITY OF BIOWASTE}

\author{
Jonathan Soto ${ }^{1 *}$, Patricia Torres ${ }^{2}$, Luz Edith Barba ${ }^{3}$, Luis Fernando Marmolejo ${ }^{4}$, Wilmar A. Torres ${ }^{5}$, \\ María A. Guzmán ${ }^{6}$, Laura M. Perea ${ }^{7}$
}

\begin{abstract}
${ }^{1^{*}}$ Ing. Sanitario y Ambiental, estudiante de M.Sc., Escuela de Ingeniería de Recursos Naturales y del Ambiente-EIDENAR. Universidad del Valle, calle 13 No 100-00, Cali, Colombia, e-mail: jonathan.soto.paz@correounivalle.edu.co; ${ }^{2}$ Ing. Sanitaria, M.Sc., Ph.D., Prof. Titular, Escuela de Ingeniería de Recursos Naturales y del Ambiente-EIDENAR. Universidad del Valle, calle 13 No 100-00, Cali, Colombia, e-mail: patricia.torres@correounivalle.edu.co; ${ }^{3}$ Ing. Sanitario, M.Sc., Ph.D., Prof. Asociado, Escuela de Ingeniería de Recursos Naturales y del Ambiente-EIDENAR. Universidad del Valle, calle 13 No 100-00, Cali, Colombia, e-mail: luis.marmolejo@correounivalle.edu.co; ${ }^{4}$ Quimica, M.Sc., Escuela de Ingeniería de Recursos Naturales y del Ambiente-EIDENAR. Universidad del Valle, calle 13 No 100-00, Cali, Colombia, e-mail: luz.barba@correounivalle.edu. co; ${ }^{5}$ Estadístico, Grupo de Investigación en Estadística Aplicada - INFERIR. Universidad del Valle, calle 13 No 100-00, Cali, Colombia, e-mail: wilmar.alexander.torres@correounivalle.edu.co; ${ }^{6}$ Estudiante de Ing. Sanitaria y Ambiental, Escuela de Ingeniería de Recursos Naturales y del Ambiente-EIDENAR. Universidad del Valle, calle 13 No 100-00, Cali, Colombia, e-mail: maria.guzman.vargas@correounivalle.edu.co; ${ }^{7}$ Estudiante de Ing. Sanitaria y Ambiental, Escuela de Ingeniería de Recursos Naturales y del Ambiente-EIDENAR. Universidad del Valle, calle 13 No 100-00, Cali, Colombia, e-mail: laura.perea@correounivalle.edu.co
\end{abstract}

Rev. U.D.C.A Act. \& Div. Cient. 18(2): 485-495, Julio-Diciembre, 2015

\section{RESUMEN}

El compostaje es una de las tecnologías más utilizadas para el aprovechamiento de los biorresiduos de origen municipal, en los países en desarrollo. Siendo un proceso aerobio, la eficiencia de la aireación puede afectar el desarrollo del proceso y la calidad del producto. En este estudio, se evaluó, a escala de laboratorio, la influencia de la concentración del oxígeno disuelto sobre la biodegradabilidad aerobia de los biorresiduos del restaurante de la Universidad del Valle, Colombia. Para ello, se estudiaron cinco concentraciones de oxígeno disuelto (menor a 0,$5 ; 1,0 ; 1,5 ; 2,0$ y $2,5 \mathrm{mg} \mathrm{L}^{-1}$ ), a través de un modelo cinético diferencial de primer orden, para determinar las tasas de utilización del sustrato, tasas de crecimiento de biomasa y por medio de respirometría, la tasa de consumo de oxígeno. Se realizaron análisis estadísticos, a partir de un modelo lineal mixto y la prueba de Tukey $(p<0,05)$, encontrándose que las concentraciones de OD evaluadas, se pueden agrupar en tres grupos, en términos reducción de $\mathrm{DQO}$ : i) OD menor a $0,5 \mathrm{mg} \mathrm{L}^{-1}$; ii) $1,0 \leq \mathrm{OD} \leq$ $1,5 \mathrm{mg} \mathrm{L}^{-1}$ y iii) $2,0 \leq \mathrm{OD} \leq 2,5 \mathrm{mg} \mathrm{L}^{-1}$, siendo este último, en el que se presentan las condiciones más favorables para la cinética de transformación del sustrato con TUS, entre 0,18 a $0,21 d^{-1}$, en las cuales, se tienen las mayores TCO y TCB.
En contraste, los grupos i y ii presentaron TUS, entre 0,02 a $0,12 \mathrm{~d}^{-1}$, lo que indica que el OD incide en la transformación del sustrato.

Palabras clave: Aprovechamiento, cinética, compostaje, residuos sólidos.

\section{SUMMARY}

Composting is one of the most used technologies for the use of municipal biowaste - MBW. Being an aerobic process, the efficiency of aeration can affect the development of the process and product quality. In this study, we evaluated at laboratory scale, the influence of the concentration of dissolved oxygenOD on aerobic biodegradability of BOM of a municipality, where segregation practices are performed at the source and selective collection; for this five concentrations of OD $(<0.5$; $1.0 ; 1.5 ; 2.0 ; 2.5 \mathrm{mg} \mathrm{L}^{-1}$ ) was studied by differential first-order kinetic model to determine rates Substrate Utilization-TUS, biomass growth rates-TCB and through respirometry the rate of oxygen consumption-TCO. Statistics from a mixed linear model analysis and Tukey test $(\mathrm{p}<0.05)$ were performed, finding that concentrations of $\mathrm{OD}$ assessed, fall into three groups in terms of COD reduction: $i$ ) less than $0.5 \mathrm{mg} \mathrm{OD}$ 
$\left.* \mathrm{~L}^{-1}\right)$; ii) $1.0 \leq \mathrm{OD} \leq 1.5 \mathrm{mg} \mathrm{L}^{-1}$ and iii) $2.0 \leq \mathrm{OD} \leq 2.5 \mathrm{mg}$ $\mathrm{L}^{-1}$, the latter in which the most favorable conditions for the transformation kinetics are presented substrate with TUS between 0,18 to $0,21 \mathrm{~d}^{-1}$, in which they have the greatest TCO and TCB. In contrast, groups $i$ and ii presented TUS between 0.02 and $0.12 \mathrm{~d}^{-1}$, indicating that the OD affects the transformation of the substrate.

Key words: Use, cynetics, composting, solid waste.

\section{INTRODUCCIÓN}

La generación creciente de los residuos sólidos municipales representa una problemática, a nivel mundial, que se ha agudizado en los últimos años. En los países desarrollados, la tasa de generación típica oscila, entre 1,43 a 2,08kg/habitante/día y en los países en desarrollo, entre 0,3 a 1,4kg/ habitante/día, de acuerdo a lo reportado por Troschinetz \& Mihelcic (2009). En estos últimos, la mayor fracción de los RSM está constituida por biorresiduos de origen municipalBOM (Hoornweg \& Bhada, 2012), lo que hace que su disposición final en rellenos sanitarios sea una práctica inadecuada, ya que agota la vida útil de estos sitios y conduce a la generación de lixiviados y de gases efecto invernadero, que tienen un impacto nocivo sobre el ambiente y la salud humana (He et al. 2011).

El compostaje es una de las alternativas de mayor aplicación para la valorización de los BOM, como lo mencionan Li et al. (2013), debido a que el producto final tiene un alto potencial de aprovechamiento agrícola, al utilizarlo como mejorador de suelos (Stentiford \& de Bertoldi, 2010); no obstante, experiencias reportadas por Barreira et al. (2006) muestran que en los países en vía de desarrollo no cumplen con la calidad requerida para su aplicación.

La concentración de oxígeno afecta la actividad de los microorganismos y, por ende, la tasa de degradación del sustrato, lo que incide en la duración de las etapas mesofilica, termofílica y de maduración, para garantizar la estabilidad y madurez del producto, además de incidir en aspectos de diseño y de operación de los procesos biológicos (Lin et al. 2008).

El desarrollo de ensayos de biodegradabilidad o respiración aerobia ha permitido estudiar, a escala de laboratorio, las condiciones, bajo las cuales, se podría mejorar el desempeño del proceso de compostaje. Estos ensayos, se clasifican en dos grandes grupos: los que se basan en la producción de dióxido de carbono y los que cuantifican el consumo de oxígeno y suministran información, tanto para el diseño como para la determinación de la concentración de oxígeno, que se requiere para llevar a cabo los procesos de oxidación, la medición indirecta de la actividad microbiana y la cinética de transformación del sustrato (Komilis, 2015).
Según Barrena et al. (2013), la respirometría puede ser utilizada para clasificar la biodegradabilidad de los residuos orgánicos en tres categorías principales, de acuerdo con la tasa de consumo de oxígeno: i) alta biodegrabilidad: mayor a $5 \mathrm{gO}_{2} \mathrm{~kg}^{-1} \mathrm{MOh}^{-1}$; ii) biodegradabilidad moderada: 2 a $5 \mathrm{gO}_{2} \mathrm{~kg}^{-1} \mathrm{MOh}^{-1}$ y iii) biodegradabilidad baja: menor que $2 \mathrm{gO}_{2}\left(\mathrm{kgMOh}^{-1}\right.$. Autores, como Richard et al. (2006), han determinado rangos de tasas de aireación para el control del proceso, que oscilan entre 0,7 a $2,8 \mathrm{mg} \mathrm{L}^{-1}$.

En este estudio, se evaluó, a escala de laboratorio, mediante ensayos respirométricos, la influencia de diferentes concentraciones de oxígeno sobre la biodegradabilidad de los BOM del restaurante universitario de la universidad del Valle, bajo la premisa de separación en la fuente y recolección selectiva.

\section{MATERIALES Y MÉTODOS}

El sustrato provino del restaurante de la universidad del Valle y fue validado con los BOM de un municipio, donde se realizan prácticas de separación en la fuente y recolección selectiva, como parte de la gestión de los residuos sólidos municipales y que ingresan a una planta de manejo de residuos sólidos. El programa de muestreo y de caracterización de sustrato correspondió a cinco muestras, de 1,0kg.

Previo a la caracterización fisicoquímica y a la realización de los ensayos biológicos, el sustrato tuvo un tratamiento, que consistió en un proceso de trituración, utilizando una licuadora CB15 Waring Commercial, para garantizar un tamaño de partícula de 5 a $7 \mathrm{~mm}$, de acuerdo con lo recomendado por Liwarska et al. (2002). Las variables medidas a las muestras de BOM fueron: $\mathrm{pH}$, alcalinidad total y bicarbonática, demanda química de oxígeno total y filtrada, demanda bioquímica de oxígeno - $\mathrm{DBO}_{5}$, sólidos totales y volátiles, carbono orgánico oxidable, nitrógeno total, amonio, fósforo, potasio, proteínas, carbohidratos, celulosa y lignina, medidas según APHA et al. (2005); estas variables también se midieron al final de los ensayos. La fracción biodegradable presente en el sustrato, se determinó mediante la ecuación 1 (Espinosa et al. 2007).

$$
\mathrm{FB}=0,83-0,028 \mathrm{CL} \quad \text { ecuación } 1
$$

Donde, FB corresponde a la fracción biodegradable en base al material volátil; 0,83 y 0,028 son constantes empíricas; CL es el contenido de lignina en porcentaje.

Por otra parte, se cuantificó el nitrógeno amoniacal no ionizado y el ión amonio presente en los BOM, de acuerdo con Sterling et al. (2001), para lo cual, se emplearon las ecuaciones 2 y 3 . 


$$
\mathrm{NH}_{3}=1,13 * 10^{-9} * \frac{\mathrm{NH}_{3}}{\mathrm{H}^{+}} \mathrm{T} \text { ecuación } 2
$$

Donde, $\mathrm{NH}_{3}$ es el nitrógeno amoniacal no ionizado; $\mathrm{NH}_{3} \mathrm{~T}$ corresponde al nitrógeno amoniacal total $\mathrm{y}^{+}$es la concentración de iones de hidrógeno.

$$
\mathrm{NH}_{3} \mathrm{~T}=\mathrm{NH}_{3}+\mathrm{NH}_{4}^{+} \text {ecuación } 3
$$

Donde, $\mathrm{NH}_{3} \mathrm{~T}$ corresponde al nitrógeno amoniacal total; $\mathrm{NH}_{3}$ es el nitrógeno amoniacal no ionizado y $\mathrm{NH}_{4}{ }^{+}$es el ion amonio.

Los ensayos, se desarrollaron a escala de laboratorio y tuvieron una duración de 264 horas. Los reactores fueron de vidrio, con un volumen de $5 \mathrm{~L}$ de capacidad y 4,2L de volumen útil, dejando un espacio libre de 0,8L, para minimizar las pérdidas del sustrato por burbujeo y agitación. Se simularon los rangos de temperatura de las fases mesofílica, termofílica y de enfriamiento, a través de un circuito eléctrico en paralelo, considerando lo reportado en la literatura (Mason \& Milke, 2005).

Con el fin de garantizar condiciones favorables en el proceso, se realizó un ajuste de $\mathrm{pH}$ al sustrato a 7,0 como una forma de mejorar el proceso de biodegradación, con una solución de $\mathrm{NaHCO}_{3}$, al 4\%. Para evaluar la influencia del nivel del $\mathrm{OD}$, se ejecutó un diseño experimental en bloques por repeticiones, en el que se evaluaron cuatro concentraciones $\left(1,0 ; 1,5 ; 2,0\right.$ y $\left.2,5 \mathrm{mg} \mathrm{L}^{-1}\right)$, que se definieron de acuerdo con la literatura y un control, al que no se le suministró oxígeno de forma mecánica y presentó una concentración inferior a $0,5 \mathrm{mg} \mathrm{L}^{-1}$.

Durante el desarrollo del proceso de digestión aerobia, las variables de medición permanentes correspondieron a la temperatura, $\mathrm{pH}$, OD y la DQO total, que permitieron monitorear el proceso, a escala de laboratorio, a fin de garantizar la reproducción de las etapas típicas, del proceso de compostaje.

Para estimar el efecto de la concentración de OD, se determinaron las tasas de degradación del Sustrato-TUS, consumo de oxígeno-TCO y de crecimiento de biomasa-TCB, para cada una de las etapas en el proceso, así como también las tasas globales. La TUS, se determinó con el método cinético diferencial, a partir de la regresión lineal de la curva del decaimiento de sustrato, en función del tiempo. Este método permite determinar el orden de reacción que se aproxima al proceso de degradación del sustrato (Ecuación 4).

$$
\underline{\mathrm{dC}}=\frac{-\mathrm{kC}^{\mathrm{n}}}{\mathrm{dt}} \text { ecuación } 4
$$

Donde, $C$ DQO para tasa de degradación del Sustrato; $k$ constante cinética; $t$ duración del ensayo; $n$ orden de la reacción.

La TCO, se determinó mediante pruebas puntuales, que se ejecutaron diariamente, durante el desarrollo de cada etapa experimental, acorde con el ensayo de disminución de la concentración de OD en el tiempo, como se presenta en la ecuación 5 (Van Haandel \& Van der Lubbe, 2007).

$$
\mathrm{TCO}=\int_{0}^{t 1} \mathrm{ConO}_{2} \mathrm{dt}
$$

Donde, TCO tasa de consumo de oxígeno; $\int_{0}^{t_{1}} \operatorname{Con} \mathrm{O}_{2} \mathrm{dt}$ : consumo de oxígeno durante la duración del ensayo.

La TCB, se estipuló considerando la degradación del sustrato y el consumo específico de oxígeno por parte de la biomasa activa en el proceso. La ecuación 6 presenta la forma en la que se cuantificó el crecimiento de biomasa, en cada etapa del proceso.

$$
\mathrm{TCB}=\frac{\int_{0}^{\mathrm{t} 1} \operatorname{Con} \mathrm{O}_{2} \mathrm{dt}}{\mathrm{DQO}} \quad \text { Ecuación } 6
$$

Donde, TCB tasa de crecimiento de biomasa; DQO degradación del sustrato.

Con la finalidad de establecer si existen diferencias significativas entre los niveles de OD, en cuanto a la biodegradabilidad del sustrato, en términos de $\mathrm{DQO}(\mathrm{T})$, se empleó un modelo lineal mixto (Verbeke \& Molenberghs, 2009), considerando las variables: tratamientos-niveles de $\mathrm{OD}$, tiempo y como variable dependiente, la cantidad de $\mathrm{DQO}$, en términos logarítmicos, con la finalidad de validar correctamente los supuestos, en los que se basa la metodología empleada. Además, se realizó la prueba de comparación múltiple de Tukey, a un nivel de significancia del 5\%; el análisis estadístico, se llevó a cabo empleando el programa estadístico $\mathrm{R}$ i386 3.0.2. Los resultados encontrados fueron comparados con estudios previos (Soto et al. 2015) efectuados con el mismo sustrato y en las mismas condiciones, con excepción del nivel de OD, que se suministró, en un nivel del orden de 5,0 $\mathrm{mg} \mathrm{L}^{-1}$.

\section{RESULTADOS Y DISCUSIÓN}

La tabla 1 presenta las características fisicoquímicas de los $\mathrm{BOM}$, que fueron utilizados como sustrato en los ensayos. Se observa, que el $\mathrm{pH}$ es ácido, asociado al aumento de la producción de ácidos orgánicos volátiles por la descomposición de los BOM, a la ausencia de ATB y a una baja AT, resultados similares a los encontrados por Li et al. (2013). Autores, como Abdulkarim \& Abdullahi (2010) y Soto et al. (2015), indican la necesidad de ajustar estas variables al inicio del proceso con un alcalinizante, que aporte capacidad 
Tabla 1. Composición física de los BOM.

\begin{tabular}{|c|c|c|}
\hline Parámetro & Unidad & Sustrato (Promedio $\pm \mathrm{Sd}$ ) \\
\hline $\mathrm{pH}$ & Unid & $5,28 \pm 0,04$ \\
\hline Alcalinidad total - AT & $\mathrm{mg} \mathrm{L}^{-1}$ & $4211,27 \pm 6,52$ \\
\hline Alcalinidad bicarbonática - ATB & $\mathrm{mg} \mathrm{L}^{-1}$ & $0,00 \pm 0,00$ \\
\hline Demanda Química de Oxígeno- DQO & $\mathrm{mg} \mathrm{L}^{-1}$ & $82050,00 \pm 378,8$ \\
\hline Demanda química de oxígeno filtrada - DQOf & $\mathrm{mg} \mathrm{L}^{-1}$ & $20528,91 \pm 1,05$ \\
\hline Demanda bioquímica de Ooxígeno-DBO 5 & $\mathrm{mg} \mathrm{L}^{-1}$ & $72394,25 \pm 0,05$ \\
\hline $\mathrm{DBO}_{5} / \mathrm{DQO}$ & -- & $0,9 \pm 0,05$ \\
\hline Sólidos totales - ST & $\mathrm{mg} \mathrm{L}^{-1}$ & $58701,00 \pm 216,47$ \\
\hline Sólidos volátiles - SV & $\mathrm{mg} \mathrm{L}^{-1}$ & $54619,00 \pm 186,26$ \\
\hline SV/ST & -- & $0,93 \pm 0.012$ \\
\hline Carbono orgánico oxidable** & $\mathrm{g} \mathrm{Kg}^{-1}$ & $26,86 \pm 11,68$ \\
\hline Nitrógeno total** & $\mathrm{mg} \mathrm{L}^{-1}$ & $1725,78 \pm 516,76$ \\
\hline Amonio & $\mathrm{mg} \mathrm{L}^{-1}$ & 258,26 \\
\hline Relación C/N & ---------- & $20,48 \pm 8,96$ \\
\hline Fósforo** & $\mathrm{mg} \mathrm{L}^{-1}$ & $391,05 \pm 0,44$ \\
\hline Potasio** & $\% \mathrm{BH}$ & $1,20 \pm 0,21$ \\
\hline Proteínas** & $\% \mathrm{BH}$ & $4,97 \pm 0,11$ \\
\hline Carbohidratos** & $\% \mathrm{BH}$ & $9,74 \pm 2,07$ \\
\hline Celulosa** & $\% \mathrm{BH}$ & $0,45 \pm 0,98$ \\
\hline Lignina** & $\% \mathrm{BH}^{-1}$ & $1,59 \pm 0,88$ \\
\hline Fracción biodegradable & ---------- & $0,78 \pm 0,88$ \\
\hline
\end{tabular}

* Parámetros realizados con 11 datos $(\mathrm{n}=11)$; ** Parámetros realizados con

5 datos $(n=5)$. BH: Porcentaje en base húmeda.

buffer y neutralice la acidez.

Se observa, en estos resultados, la predominancia orgánica, reflejada en las relaciones $\mathrm{DBO}_{5} / \mathrm{DQO}$ y SV/ST, que también muestran el alto potencial de degradación biológica de los BOM. Aunque el contenido de carbono en el sustrato es inferior al reportado para BOM, el nitrógeno se halla próximo a lo expuesto por autores, como lqbal et al. (2010). La forma predominante del nitrogeno corresponde al ión amonio, mientras que el nitrogeno total amoniacal $\left(\mathrm{NH}_{3}\right)$, se encontró en una concentracion de $0,034 \mathrm{mg} \mathrm{L}^{-1}$, lo que indica que, de presentarse una inhibicion por el $\mathrm{pH}$, ésta será baja. Por otra parte, la relacion $\mathrm{C} / \mathrm{N}$, se registra en el rango sugerido por Neklyudov et al. (2008), de 20 a 30; sin embargo, investigadores, como Tognetti et al. (2007) han mostrado que el proceso se puede realizar hasta con una relación, incluso, del orden de 15, mientras que Diaz \& Savage (2007) consideran una relación adecuada, entre 25 a 30.

El contenido de fósforo es mayor al reportado por Iqbal et al. (2010) e inferior al sugerido por Neklyudov et al. (2008); no obstante, la relacion DQO:N:P del BOM 100:2,1:0,5 es menor a la recomendada para procesos biológicos aerobios (Torres, 2013), lo que puede indicar dificultad para lograr la biodegradacion total del sustrato. Otros compuestos, como las proteínas y los carbohidratos, se encontraron en niveles similares a los reportados por Chen et al. (2010). Estos son de gran importancia en el proceso, debido a que son utilizados, inicialmente, por los consorcios microbianos, que inician con la degradacion de estos compuestos (Chiumenti et al. 2005). Por otra parte, los contenidos de celulosa y de lignina fueron inferiores a los obtenidos por Chen et al. (2010); de esta manera, la FB equivalente a 0,78 , ratifica la potencial biodegradación del BOM.

Con relación al monitoreo del proceso (Figura 1), la temperatura presentó un comportamiento típico del proceso de compostaje, con ocurrencia de las etapas mesofílica y ter- 
mofílica, en tiempos recomendados por autores, como Neklyudov et al. (2008) y con un descenso de la temperatura, acorde con la fase de enfriamiento; no obstante, según lo reportado por de Guardia et al. (2008), no se logró replicar la etapa de maduración, debido a la transformación del sustrato a dióxido de carbono y al volumen de los reactores, lo que limitó la duración de esta etapa. Respecto al pH, todos los reactores iniciaron el proceso con un valor ajustado a 7,0 , que fue variando en función de la temperatura y de la concentración de OD (Yun et al. 2005). En la figura 1b, se observa que a las 24 horas, el $\mathrm{pH}$ se redujo a valores del orden de 5,0, resultado asociado a la composición fácilmente acidificable del BOM, que hace que la materia orgánica sea

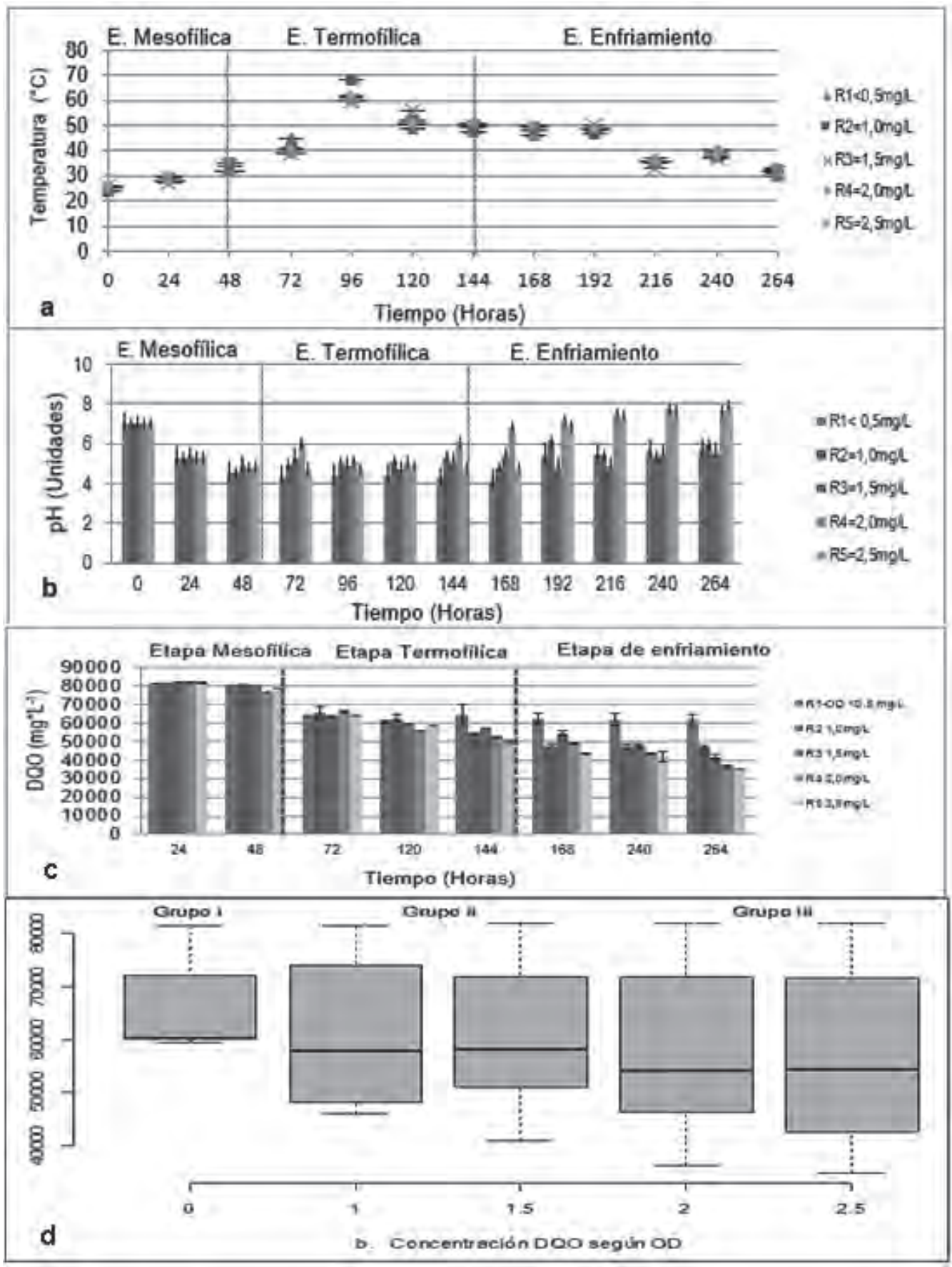

Figura 1. a) Comportamiento de la temperatura durante el ensayo; b) Valores de pH obtenidos para cada tratamiento; c) Reducción de la DQO en el tiempo; d) Variación de la DQO según los niveles de OD. 
rápidamente transformada a ácidos orgánicos (Chiumenti et al. 2005).

El efecto de la reducción del pH fue más notorio en los reactores con concentraciones de OD inferiores a 2,0 $\mathrm{mg} \mathrm{L}^{-1}$, ya que, según reportan Diaz \& Savage (2007), la concentración de oxígeno no debe ser inferior a un $10 \%$, para impedir la presencia de zonas anaerobias que puedan afectar los consorcios microbianos aerobios. Lo anterior coincide con lo reportado por Rasapoor et al. (2009), quienes concluyen que cuando se consume el oxígeno disponible y éste es limitante, se da una oxidación incompleta del sustrato, que favorece la producción de ácidos orgánicos de cadena corta y como consecuencia, se presenta un descenso del $\mathrm{pH}$.

Por otra parte, en los reactores con condición igual o superior a 2,0 $\mathrm{mg} \mathrm{L}^{-1}$, el $\mathrm{pH}$ incrementó a valores superiores a 7,0 , por la transformación del nitrógeno orgánico presente a amoniaco y por la formación de $\mathrm{CO}_{2}$, el cual, es liberado al ambiente, producto de la degradación del sustrato (Rasapoor et al. 2009). Esto coincide con lo encontrado por Soto et al. (2015), en un ensayo de biodegradabilidad aerobia, con una concentración de oxígeno del orden de 5,0 $\mathrm{mg} \mathrm{L}^{-1}$.

Evaluación del OD en el proceso. La figura 1c muestra la variación de la concentración del sustrato, medida como $\mathrm{DQO}_{(\mathrm{T})}$, durante el proceso, para cada uno de los niveles de OD estudiados, resaltándose que la mayor reducción de la $\mathrm{DQO}$, se presenta con las concentraciones de OD equivalentes a 2,0 y 2,5 $\mathrm{m} \mathrm{L} \mathrm{L}^{-1}$, coincidiendo con lo reportado por Yun et al. (2005). Por otra parte, en la figura 1d, se destaca, desde el punto de vista estadístico, la conformación de tres grupos respecto la concentración de OD, evaluadas a partir de los resultados obtenidos con el modelo lineal mixto: i) $\mathrm{OD} \leq 0,5 \mathrm{mg}$ $\mathrm{L}^{-1}$; ii) $1,0 \leq \mathrm{OD} \leq 1,5 \mathrm{mg} \mathrm{L}^{-1}$ y iii) $2,0 \leq \mathrm{OD} \leq 2,5 \mathrm{mg} \mathrm{L}^{-1}$, mostrando que las reducciones del sustrato son similares al interior de cada grupo y diferentes respecto al primero. Adicionalmente, la prueba de Tukey $(\mathrm{p}<0.05)$, mostró que el grupo con concentraciones iguales o mayores a $2,0 \mathrm{mg} \mathrm{L}^{-1}$ corresponde a los mejores tratamientos, a través de los cuales, se puede obtener una mayor biodegradación del sustrato.

En la tabla 2, se presentan las TUS, TCO y TCB determinadas para cada una de las concentraciones de OD evaluadas en los reactores y para las diferentes etapas del proceso, encontrándose que el sustrato presentó una cinética de biodegradación de orden uno, resultado similar al reportado por Liwarska et al. (2002).

Con relación a la influencia del OD, una mayor concentración intensifica la actividad de los microorganismos y, consecuentemente, estimula el consumo del sustrato, aunque su exceso incrementa las pérdidas de amonio y también afecta el proceso. Ésto se presentó en el grupo iii, donde se ob- tuvieron mayores TUS, lo que coincide con otros estudios (Guo et al. 2012), que reportan la mayor velocidad de biodegradación con concentraciones de OD, en el rango de 2,0 a $2,8 \mathrm{mg} \mathrm{L}^{-1}$.

La operación con estas concentraciones de OD fueron similares a las encontradas, con un exceso de OD del orden de 5,0 $\mathrm{mg} \mathrm{L}^{-1} \mathrm{y}$ sin adición de nutrientes, en un ensayo previo, adelantado por Soto et al. (2015). Además, se obtuvo valores de $\mathrm{pH}$ cercanos a la neutralidad, cuando el nivel de OD no excede los $2,5 \mathrm{mg} \mathrm{L}^{-1}$, en comparación con aquellos que exceden esta condición, donde los valores de $\mathrm{pH}$ son básicos afectando, posiblemente, la calidad del producto, al ser fitotóxico.

Con respecto a la TCO, hay una relación directa con la TUS y, por consiguiente, con la TCB, siendo, ésta última, donde se presentan los mayores picos de respiración, para el grupo iii y, los más bajos, para i y ii, respectivamente, lo cual, está asociado a la disponibilidad de oxígeno para la oxidación del sustrato y parte de la energía liberada, en el rompimiento de enlaces, que se utilizó para la formación de nuevo material celular, lo que permitió tener una mayor velocidad de crecimiento de biomasa. En contraste, en los reactores, con una concentración menor a 2,0 $\mathrm{mg} \mathrm{L}^{-1}$, se logró favorecer la formación de zonas anaerobias, que se encuentran relacionadas con los valores $\mathrm{pH}$ obtenidos.

Por lo anterior, los resultados encontrados con la TCO muestran que el sustrato es de alta biodegrabilidad, según la escala reportada por Barrena et al. (2013). Acorde con la TCO de cada reactor y con lo descrito por investigadores, como Adani et al. (2005), los reactores R4 y R5 presentan un índice de respiración aproximado, de 1154 y $1270 \mathrm{mgO}_{2} \mathrm{~kg}^{-1}$ de $\mathrm{BOM}$, por lo que se requieren alrededor de 144 y 168 horas, para que el producto sea estable $\left(500 \mathrm{mgO}_{2} \mathrm{~kg}^{-1}\right) \mathrm{y}$, para los demás reactores, se necesitan, al menos, 360 horas adicionales. Este resultado es análogo a lo divulgado por Oviedo et al. (2014), quienes reportan que la tasa de aireación ejerce un efecto directo sobre el proceso de biodegradación, disminuyendo el tiempo requerido para la estabilización y la madurez del compost, a escala real.

Uno de los factores que pudo incidir en la biodegradación del sustrato corresponde a la limitación de los nutrientes presentes en el sustrato, tanto de nitrógeno como de fósforo total, lo cual, pudo incidir en la formación de las estructuras celulares y, por consiguiente, de la generación de nueva biomasa, afectando adversamente el proceso.

Con relación a cada una de las etapas del proceso, se encontró que la etapa termofílica es la que rige el proceso, dada la influencia de la temperatura sobre la cinética, incrementado la degradación del sustrato (Kumar et al. 2010), ya que 


\begin{tabular}{|c|c|c|c|c|c|c|}
\hline \multirow{4}{*}{ 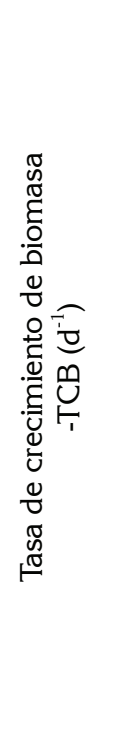 } & $\begin{array}{l}\bar{\pi} \\
0 \\
0 \\
0 \\
0 \\
b\end{array}$ & $\begin{array}{l}0 \\
0 \\
0 \\
0 \\
+1 \\
m \\
0 \\
0 \\
0\end{array}$ & 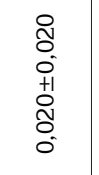 & \begin{tabular}{l}
0 \\
0 \\
0 \\
+1 \\
\multirow{0}{0}{} \\
0 \\
0
\end{tabular} & 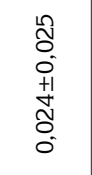 & $\begin{array}{l}\stackrel{\sim}{\tilde{N}} \\
0 \\
0 \\
+1 \\
\tilde{O} \\
0 \\
0\end{array}$ \\
\hline & 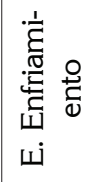 & $\begin{array}{l}\Delta \\
0 \\
0 \\
0 \\
+1 \\
0 \\
m \\
0 \\
0\end{array}$ & $\begin{array}{l}n \\
0 \\
0 \\
0 \\
+1 \\
0 \\
0 \\
0 \\
0\end{array}$ & $\begin{array}{l}\infty \\
0 \\
0 \\
0 \\
+1 \\
0 \\
\vdots \\
0 \\
0\end{array}$ & 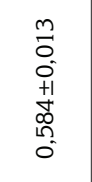 & $\begin{array}{l}\tilde{N} \\
0 \\
0 \\
+1 \\
0 \\
9 \\
0 \\
0\end{array}$ \\
\hline & 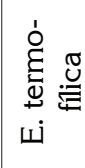 & 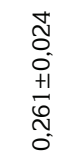 & $\begin{array}{l}\hat{N} \\
0 \\
0 \\
+1 \\
\infty \\
0 \\
0 \\
0\end{array}$ & 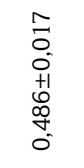 & $\begin{array}{l}8 \\
8 \\
0 \\
+1 \\
0 \\
0 \\
0\end{array}$ & $\begin{array}{l}\cong \\
0 \\
0 \\
+1 \\
\infty \\
0 \\
0 \\
0\end{array}$ \\
\hline & 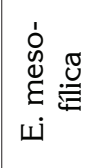 & $\begin{array}{l}\tilde{N} \\
0 \\
0 \\
+1 \\
\stackrel{1}{+} \\
\stackrel{0}{0} \\
0\end{array}$ & 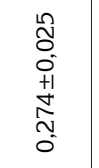 & $\begin{array}{l}0 \\
0 \\
0 \\
+1 \\
+1 \\
0 \\
0 \\
0 \\
0\end{array}$ & $\begin{array}{l}0 \\
0 \\
0 \\
0 \\
+1 \\
0 \\
0 \\
0 \\
0\end{array}$ & $\begin{array}{l}n \\
0 \\
0 \\
+1 \\
0 \\
0 \\
0 \\
0\end{array}$ \\
\hline \multirow{3}{*}{ 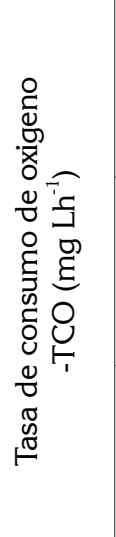 } & 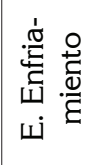 & $\begin{array}{l}\stackrel{g}{J} \\
\stackrel{-}{m} \\
\stackrel{1}{0} \\
m \\
m\end{array}$ & 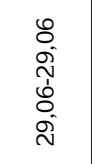 & 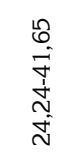 & 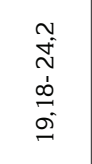 & 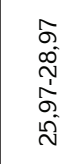 \\
\hline & 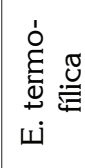 & $\begin{array}{l}\hat{\imath} \\
\text { mे } \\
\stackrel{N}{m} \\
m\end{array}$ & 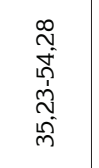 & \begin{tabular}{l}
$\infty$ \\
0 \\
N \\
$\hat{N}$ \\
$\stackrel{N}{N}$ \\
\multirow{J}{*}{}
\end{tabular} & 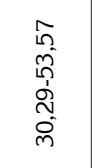 & 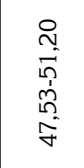 \\
\hline & 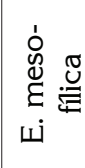 & $\begin{array}{l}\stackrel{0}{1} \\
\tilde{m} \\
\stackrel{0}{0} \\
0\end{array}$ & $\begin{array}{l}\vec{J} \\
\vec{N} \\
\tilde{N} \\
\tilde{\sim}\end{array}$ & 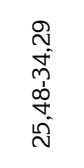 & $\begin{array}{l}m \\
\infty \\
m \\
m \\
\hat{\omega} \\
\tilde{m} \\
\tilde{m}\end{array}$ & 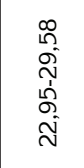 \\
\hline \multirow{4}{*}{ 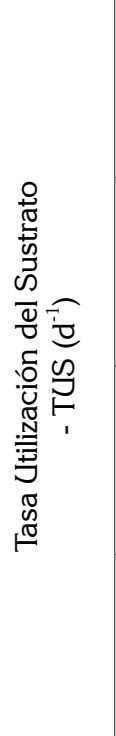 } & 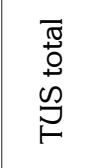 & $\begin{array}{l}\widetilde{0} \\
0 \\
0 \\
+1 \\
0 \\
0 \\
0 \\
0\end{array}$ & $\begin{array}{l}\infty \\
0 \\
0 \\
+1 \\
0 \\
0 \\
0\end{array}$ & 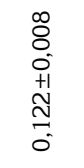 & $\begin{array}{l}\overline{8} \\
0 \\
0 \\
+1 \\
0 \\
0 \\
0 \\
0\end{array}$ & $\begin{array}{l}\overrightarrow{0} \\
0 \\
+1 \\
m \\
\vec{N} \\
0\end{array}$ \\
\hline & 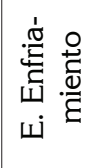 & $\begin{array}{l}0 \\
0 \\
0 \\
+1 \\
+1 \\
0 \\
0 \\
0 \\
0\end{array}$ & $\begin{array}{l}\hat{0} \\
0 \\
0 \\
+1 \\
o \\
00 \\
0 \\
0\end{array}$ & $\begin{array}{l}0 \\
8 \\
0 \\
+1 \\
0 \\
0 \\
0 \\
0\end{array}$ & $\begin{array}{l}\infty \\
8 \\
0 \\
+1 \\
01 \\
0 \\
0 \\
0\end{array}$ & $\begin{array}{l}n \\
0 \\
0 \\
+1 \\
0 \\
0 \\
0 \\
0\end{array}$ \\
\hline & 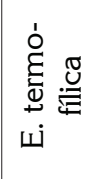 & $\begin{array}{l}n \\
0 \\
0 \\
+1 \\
0 \\
0 \\
0 \\
0\end{array}$ & $\begin{array}{l}0 \\
0 \\
0 \\
+1 \\
+1 \\
0 \\
0 \\
0\end{array}$ & $\begin{array}{l}\stackrel{m}{0} \\
0 \\
0 \\
\stackrel{1}{N} \\
\cong \\
0 \\
0\end{array}$ & $\begin{array}{l}\widetilde{0} \\
0 \\
0 \\
+1 \\
0 \\
0 \\
0\end{array}$ & $\begin{array}{l}\tilde{0} \\
8 \\
0 \\
+1 \\
0 \\
\stackrel{0}{0} \\
0\end{array}$ \\
\hline & 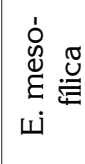 & $\begin{array}{l}\text { ב̃ } \\
0 \\
0 \\
+1 \\
0 \\
0 \\
0\end{array}$ & 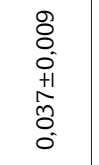 & $\begin{array}{l}8 \\
8 \\
0 \\
+1 \\
\stackrel{0}{0} \\
0 \\
0 \\
0\end{array}$ & \begin{tabular}{l}
+ \\
8 \\
0 \\
+1 \\
+1 \\
\multirow{2}{*}{} \\
0
\end{tabular} & $\begin{array}{l}\infty \\
8 \\
0 \\
+1 \\
+1 \\
\stackrel{+}{+} \\
0\end{array}$ \\
\hline $\begin{array}{l}\mathbb{0} \\
\mathbb{\infty} \\
\mathbb{1}\end{array}$ & 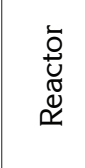 & 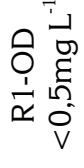 & 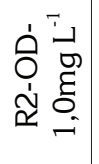 & 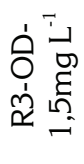 & 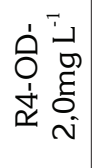 & 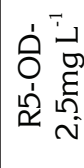 \\
\hline
\end{tabular}




\begin{tabular}{|c|c|c|c|c|c|c|c|c|c|c|c|c|c|}
\hline 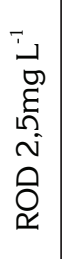 & \begin{tabular}{l}
$m$ \\
0 \\
0 \\
+1 \\
\multirow{+}{+}{} \\
$\infty$ \\
$\infty$
\end{tabular} & 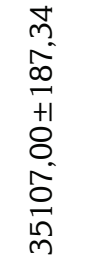 & 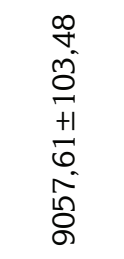 & $\begin{array}{l}1 \\
\infty \\
10 \\
m \\
+1 \\
+1 \\
0 \\
0 \\
0 \\
0 \\
0\end{array}$ & 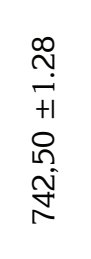 & $\begin{array}{l}\text { N } \\
\text { N } \\
+1 \\
8 \\
8 \\
\text { g }\end{array}$ & 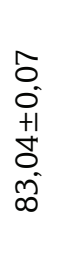 & $\begin{array}{l}\infty \\
0 \\
0 \\
+1 \\
0 \\
0 \\
\stackrel{1}{n}\end{array}$ & $\begin{array}{l}n \\
\stackrel{0}{0} \\
+1 \\
\infty \\
0 \\
-\end{array}$ & $\begin{array}{l}8 \\
0 \\
0 \\
+1 \\
1 \\
m \\
0 \\
0\end{array}$ & 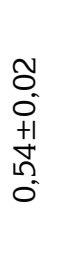 & $\begin{array}{l}\tilde{0} \\
0 \\
+1 \\
+ \\
+ \\
0\end{array}$ & \begin{tabular}{l}
5 \\
0 \\
0 \\
+1 \\
o \\
\multirow{+}{*}{}
\end{tabular} \\
\hline 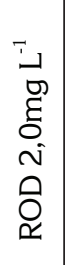 & 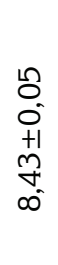 & 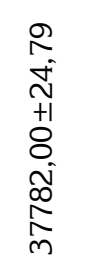 & 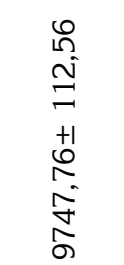 & 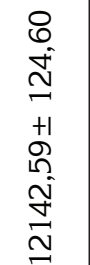 & 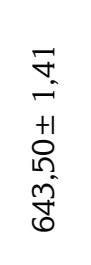 & $\begin{array}{l}\text { ㄱ } \\
\tilde{N} \\
+1 \\
8 \\
8 \\
\infty \\
0 \\
0\end{array}$ & $\begin{array}{l}\hat{\infty} \\
\infty \\
0 \\
+1 \\
m \\
\infty \\
n \\
n\end{array}$ & $\begin{array}{l}\nabla \\
0 \\
0 \\
+1 \\
\sigma \\
\sigma \\
\text { ம. }\end{array}$ & 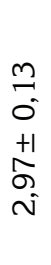 & \begin{tabular}{l}
\multirow{0}{0}{} \\
0 \\
+1 \\
+1 \\
0 \\
0
\end{tabular} & $\begin{array}{l}\tilde{O} \\
0 \\
0 \\
+1 \\
0 \\
0 \\
0\end{array}$ & $\begin{array}{l}\sigma \\
0 \\
0 \\
+1 \\
+ \\
+ \\
0\end{array}$ & $\begin{array}{l}5 \\
0 \\
0 \\
+1 \\
\text { o } \\
\text { + }\end{array}$ \\
\hline 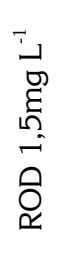 & $\begin{array}{l}\infty \\
0 \\
0 \\
+1 \\
\infty \\
0 \\
1\end{array}$ & 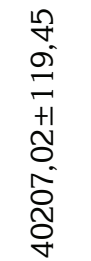 & 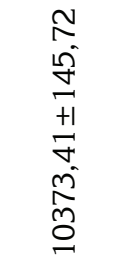 & 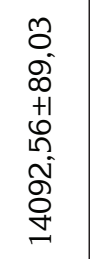 & 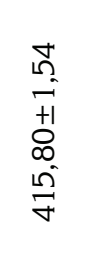 & 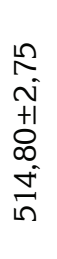 & 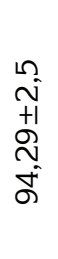 & $\begin{array}{l}\text { N } \\
\text { O } \\
+1 \\
+1 \\
\infty \\
0\end{array}$ & 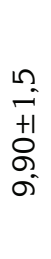 & 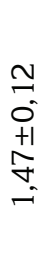 & $\begin{array}{l}\tilde{0} \\
0 \\
+1 \\
\infty \\
0 \\
\approx \\
-\end{array}$ & \begin{tabular}{l}
$\sigma$ \\
0 \\
0 \\
+1 \\
\multirow{1}{*}{} \\
0
\end{tabular} & \begin{tabular}{l}
$\sigma$ \\
0 \\
0 \\
+1 \\
Oे \\
\multirow{-}{*}{}
\end{tabular} \\
\hline 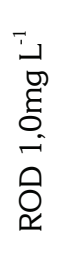 & 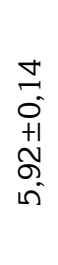 & 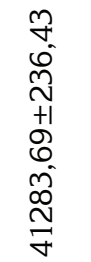 & 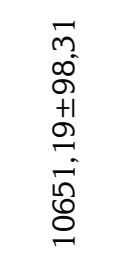 & 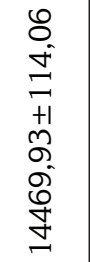 & 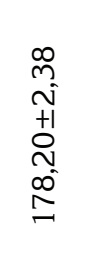 & 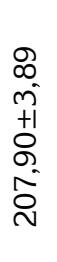 & $\begin{array}{l}\infty \\
\underset{n}{n} \\
+1 \\
\underset{n}{N}\end{array}$ & $\begin{array}{l}\overline{1} \\
0 \\
0 \\
+1 \\
0 \\
0 \\
0 \\
0\end{array}$ & $\begin{array}{l}\hat{F} \\
\hat{+1} \\
\stackrel{8}{8} \\
\dot{\sigma}\end{array}$ & $\begin{array}{l}6 \\
0 \\
0 \\
+1 \\
0 \\
m \\
\end{array}$ & $\begin{array}{l}0 \\
0 \\
0 \\
+1 \\
\stackrel{+}{\sim} \\
\end{array}$ & \begin{tabular}{l}
5 \\
0 \\
0 \\
+1 \\
\multirow{+}{*}{} \\
0
\end{tabular} & $\begin{array}{l}\sigma \\
0 \\
0 \\
+1 \\
\text { N } \\
\end{array}$ \\
\hline 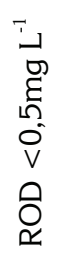 & $\begin{array}{c}\sim \\
0 \\
0 \\
+1 \\
0 \\
\dot{+} \\
\text { m. }\end{array}$ & 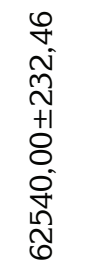 & 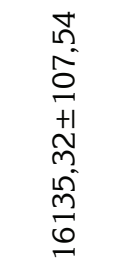 & 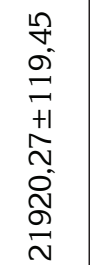 & 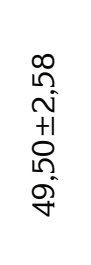 & 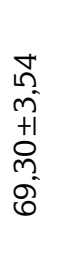 & 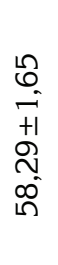 & 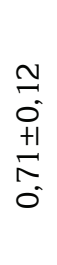 & 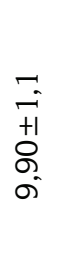 & $\begin{array}{l}\text { J } \\
0 \\
0 \\
+1 \\
0 \\
\text { m. }\end{array}$ & 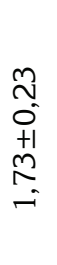 & \begin{tabular}{l}
$\sigma$ \\
0 \\
0 \\
+1 \\
\multirow{+}{*}{} \\
0
\end{tabular} & $\begin{array}{l}\sigma \\
0 \\
0 \\
+1 \\
N \\
\tilde{N} \\
-\end{array}$ \\
\hline 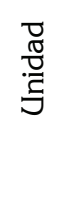 & 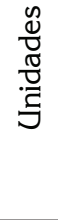 & 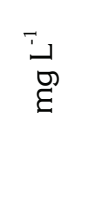 & 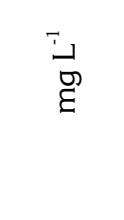 & 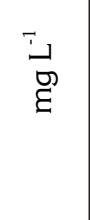 & 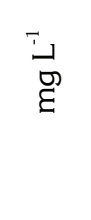 & בَّ & 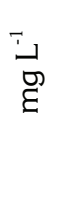 & 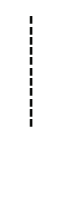 & 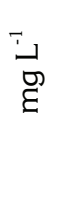 & $\begin{array}{l}T \\
\frac{T}{80} \\
\text { Do }\end{array}$ & $\begin{array}{l}5 \\
0\end{array}$ & $\begin{array}{l}I \\
\frac{1}{0} \\
\Delta \circ\end{array}$ & $\begin{array}{l}I \\
\frac{T}{80} \\
\text { so }\end{array}$ \\
\hline 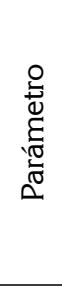 & $\frac{I}{\Omega}$ & 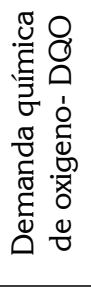 & 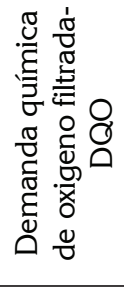 & 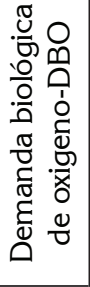 & 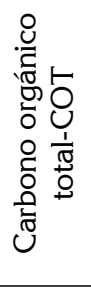 & 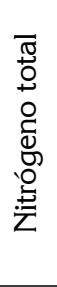 & 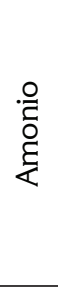 & $\begin{array}{l}Z \\
U \\
\frac{\sigma}{U} \\
\frac{0}{\mathbb{U}} \\
\mathbb{2}\end{array}$ & $\begin{array}{l}\widehat{0} \\
0 \\
\text { o } \\
\frac{0}{n} \\
\text { i }\end{array}$ & 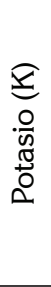 & 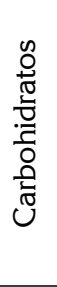 & $\begin{array}{l}\mathscr{D} \\
\frac{0}{3} \\
\frac{\mathcal{U}}{U}\end{array}$ & 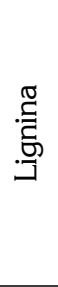 \\
\hline
\end{tabular}


temperaturas, entre 45 y $55^{\circ} \mathrm{C}$, maximizan la tasa de biodegradación (Tang et al. 2007), donde se presentan las mayores TUS, TCO y TCB (tabla 2) y un crecimiento exponencial de biomasa, como se evidencia con la TCB. Por otra parte, tanto la etapa mesofílica como de enfriamiento, presentaron TUS menores, a la percibidas en la etapa termofilica, lo que se asocia a que está estrictamente determinada por los constituyentes del sustrato, dadas las diferentes tasas de velocidad (Liwarska et al. 2002).

En la tabla 3, se resumen los resultados encontrados para ambas concentraciones, al final del ensayo.

El pH al final del ensayo, como consecuencia de las diferentes concentraciones de OD estudiadas, muestra que para el grupo iii, los valores son básicos, debido a la dinámica de transformación del nitrógeno, por la formación del amoniaco (de Guardia et al. 2008). En contraste, en los reactores con concentraciones de OD de los grupos i y ii, se observan valores ácidos, lo cual, indica que la concentración de OD afecta el proceso, al igual que cuando se excede la concentración de OD, como lo describe Soto et al. (2015). El producto, como se muestra en la tabla 3, muestra un contenido de materia orgánica, que se evidencia con relaciones de $\mathrm{DBO}_{5} / \mathrm{DQO}$, entre 0,31 y 0,35. Esto también se asocia a la presencia de material de lenta biodegradación, como la lignina y la celulosa, cuya biodegradación no ocurre a una misma tasa. Por otra parte, las relación C/N, para el grupo iii, es similar a las reportadas en estudios realizados por Gómez et al. (2015).

En todos los grupos, se evidenció un bajo contenido de fósforo, lo que reduce las posibilidades de tener un proceso eficiente, debido a las potenciales limitaciones en los procesos metabólicos esenciales, como el crecimiento, la síntesis celular y los requerimientos de energía, que se realizan a partir de este macronutriente, precisando mejorar las características fisicoquímicas, en términos de nutrientes del sustrato, a través de alternativas, como el co-compostaje, que permita tratar diferentes tipos de residuos, con la finalidad de darle un valor agregado, cerrar ciclos y minimizar la contaminación. Con relación al potasio, se encontró que su elevado contenido es acorde con lo reportado por Oviedo et al. (2014) y se asocia a las cáscaras de plátano, presentes en el BOM.

Analizados los resultados, se concluye que los BOM se caracterizan por presentar un elevado contenido de materia orgánica y la simulación, a escala de laboratorio, del proceso de compostaje, es viable. En esta investigación, se evidenció la necesidad de prolongar el ensayo a tiempos superiores a 15 días. Adicionalmente, se probó que concentraciones de OD, entre 2,0 y 2,5mg/L, generan condiciones óptimas para la biodegradabilidad aerobia de los BOM, favoreciendo la actividad microbiológica, durante cada una de las etapas estudiadas del proceso y la consecución de un material más estable, al terminar la etapa de enfriamiento; de igual forma, fue posible determinar que concentraciones de OD, por debajo de $1,5 \mathrm{mg} \mathrm{L}^{-1}$, limitan, considerablemente, la actividad biológica, durante la etapa termofílica, impidiendo, de esta forma, la utilización del sustrato y la estabilización del material obtenido, al terminar el proceso.

Agradecimientos: Los autores agradecen al Departamento de Ciencia Tecnología e investigación COLCIENCIAS, por la beca de Jóvenes Investigadores Convocatoria 2014, del profesional Jonathan Soto Paz y a la Universidad del Valle. Conflictos de intereses: El manuscrito fue preparado y revisado con la participación de todos los autores, quienes declaramos que no existe conflicto de intereses que ponga en riesgo la validez de los resultados presentados. Financiación: Este estudio fue financiado por la Universidad del Valle, en el proyecto con Cl 2811 "Aprovechamiento de subproductos a partir de la digestión biológica de residuos sólidos de origen municipal".

\section{BIBLIOGRAFÍA}

1. ABDULKARIM, B.; ABDULLAHI, M. 2010. Effect of buffer (NaHCO3) and waste type in high solid thermophilic anaerobic digestion. Int. J. Chem Tech. Res. 2(2):980.984.

2. ADANI, F.; UBBIALLI, C.; GENERINI, P. 2005. The determination of biological stability o composts using the dynamic respiration index: the results of experience after two years. Waste Managem. 26:41-48.

3. APHA; AWWA; WEF. 2005. Standard methods for the examination of water wastewater. 21 ed. Washington DC: American Public Health Association, American Water Works Association, Water Environ.

4. BARREIRA, L.; PHILIPPI, A.; RODRIGUES, M. 2006. Usinas de compostagem do Estado de São Paulo: qualidade dos compostos e processos de produção. Eng. Sanit. Ambient. 11:385-393.

5. BARRENA, R.; ARTOLA, A.; FONT, X.; GEA, T.; POGNANI, M.; PONSÁ, S.; COLÓN, J.; RUGGIERI, L.; SÁNCHEZ, A. 2013. La respirometría como herramienta para la clasificación de la biodegradabilidad de residuos orgánicos. Resultados de la experiencia de 10 años. Grupo e Investigación en ompostaje, Departamento de Ingeniería Química, Universitad Autònoma e Barcelona. España. p.12-19. 
6. CHEN, X.; ROMANO, R.T; ZHANG, R. 2010. Anaerobic digestion of food wastes for biogas production. Agric. Biol. Eng. 3:61-71.

7. CHIUMENTI, A.; CHIUMENTI, R.; DÍAZ, L.; SAVAGE, G.; EGGERTH, L.; GOLDSTEIN, N. 2005. Modern composting technologies. BioCycle-JG Press, Emmaus, PA. 12:1774-1782.

8. DE GUARDIA, A.; PETIOT, C.; ROGEAU, D. 2008. Influence of aeration rate and biodegradability fractionation on composting kinetics. Waste Managem. 28:73-84.

9. DIAZ, L.; SAVAGE, G. 2007. Chapter 4. Factors that affect the process. Waste Managem. 8:49-65.

10. ESPINOSA, L.; LÓPEZ, T.; PELLÓN, A.; MAYARÍ, N.; FERNÁNDEZ, C.A. 2007. La fracción orgánica de los residuos sólidos urbanos como fuente potencial de producción de biogás. Cienc. Biol. 38:33-37.

11. GÓMEZ, M.; JUÁREZ, M. INSAM, H. 2015. Merging two waste streams, wood ash and biowaste, results in improved composting process and end products. Science Total Environm. 511:91-100.

12. GUO, R.; LI, G.; JIANG, T.; SCHUCHARDT, F.; CHEN, T.; ZHAO, Y.; SHEN, Y. 2012. Effect of aeration rate, C/N ratio and moisture content on the stability and maturity of compost. Bioresource Techn. 112:171-178.

13. HE, L.; HUANG, G.; LU, H. 2011. Greenhouse gas emissions control in integrated municipal solid waste management through mixed integer bilevel decisionmaking. Hazard. Mat. 193:112-119.

14. HOORNWEG, D.; BHADA, P. 2012. What a waste: Waste management around the world. Washington, DC: World Bank. p.9-15.

15. IQBAL, M.K.; SHAFIQ, T.; HUSSAIN, A.; AHMED, K. 2010. Effect of enrichment on chemical properties of MSW compost. Biores. Techn. 101:5969-5977.

16. KOMILIS, D.P. 2015. Compost quality: Is research still needed to assess it or do we have enough knowledge? Waste Managem. 38:1-2.

17. KUMAR, M.; OU, Y.-L.; LIN, J.-G. 2010. Co-composting of green waste and food waste at low $\mathrm{C} / \mathrm{N}$ ratio. Waste Managem. 30:602-609.
18. LI, Z.; LU, H.; REN, L.; HE, L. 2013. Experimental and modeling approaches for food waste composting: A review. Chemosphere. 93:1247-1257.

19. LIN, Y.; HUANG, G.; LU, H.; HE, L. 2008. Modeling of substrate degradation and oxygen consumption in waste composting processes. Waste Managem. 28:1375-1385.

20. LIWARSKA, E.; BIZUKOJC, M.; LEDAKOWICZ, S. 2002. Kinetics of the aerobic biological degradation of shredded municipal solid waste in liquid phase. Water Res. 36:2124-2132.

21. MASON, I.; MILKE, M. 2005. Physical modelling of the composting environment: A review. Part 1: Reactor systems. Waste Managem. 25:481-500.

22. NEKLYUDOV, A.; FEDOTOV, G.; IVANKIN, A. 2008. Intensification of composting processes by aerobic microorganisms: A review. Applied Biochem. Microb. 44:6-18.

23. OVIEDO, E.; MARMOLEJO REBELLÓN, L.; TORRES LOZADA, P. 2014. Influencia de la frecuencia de volteo para el control de la humedad de los sustratos en el compostaje de biorresiduos de origen municipal. Rev. Int. Contam. Ambiental. 30:91-100.

24. RASAPOOR, M.; NASRABADI, T.; KAMALI, M.; HOVEIDI, H. 2009. The effects of aeration rate on generated compost quality, using aerated static pile method. Waste Managem. 29:570-573.

25. RICHARD, T.; WALKER, L.; GOSSETT, J. 2006. Effects of oxygen on aerobic solid-state biodegradation kinetics. Biotechnol. Progress. 22:60-69.

26. SOTO, J.; TORRES, P.; OVIEDO, R.; MARMOLEJO, L; ZAMBRANO, P. 2015. Influence of $\mathrm{pH}$ and nutrients on the aerobic biodegradability of municipal biowastes. Rev. AFINIDAD. 72:572-585.

27. STENTIFORD, E.; DE BERTOLDI, M. 2010. Composting process. En: Cristensen, T. (ed.) Solid Waste Technology and Management. Vol 1-2. Blackwell Publ. Ltd. 1052p.

28. STERLING, Jr.; LACEY, R.; ENGLER, C.; RICKE, S. 2001. Effects of ammonia nitrogen on $\mathrm{H} 2$ and $\mathrm{CH} 4$ production during anaerobic digestion of dairy cattle manure. Biores. Tech. 77:9-18. 
29. TANG, J.; SHIBATA, A.; ZHOU, Q.; KATAYAMA, A. 2007. Effect of temperature on reaction rate and microbial community in composting of cattle manure with rice straw. J. Biosci. Bioeng. 104:321-328.

30. TOGNETTI, C.; MAZZARINO, M.; LAOS, F. 2007. Improving the quality of municipal organic waste compost. Biores. Techn. 98:1067-1076.

31. TORRES, P. 2013. Perspectivas del tratamiento anaerobio de aguas residuales domésticas en países en desarrollo. Rev. EIA. 9:115-129.

32. TROSCHINETZ, A.; MIHELCIC, J. 2009. Sustainable recycling of municipal solid waste in developing countries. Waste Managem. 29:915-923.
33. VAN HAANDEL, A.; VAN DER LUBBE, J. 2007. Handbook biological waste water treatment. Quist Publ., Leidschendam, The Netherlands. 570p.

34. VERBEKE, G.; MOLENBERGHS, G. 2009. Linear mixed models for longitudinal data, Springer Sc \& Business Media. p.1-12

35. YUN, Y.S.; PARK, J.I.; PARK, J.M. 2005. High-rate slurryphase decomposition of food wastes: indirect performance estimation from dissolved oxygen. Process Biochem. 40:1301-1306.

Recibido: Junio 23 de 2015

Aceptado: Septiembre25 de 2015

\section{Cómo citar:}

Soto, J.; Torres, P.; Barba, L.E.; Marmolejo, L.F.; Torres, W.A.; Guzmán, M.A.; Perea, L.M. 2015. Efecto del oxígeno disuelto sobre la biodegradabilidad de biorresiduos. Rev. U.D.C.A Act. \& Div. Cient. 18(2): 485-495. 